\title{
Response
}

\section{Response to Pachter and Song's comments}

\author{
Laboratory Investigation (2005) 85, 1451-1452. doi:10.1038/labinvest.3700334
}

In reply: To answer to the 'letter-to-the-editor' addressed by Dr Pachter in response to the article by Coisne et al: 'Mouse syngenic in vitro blood-brainbarrier model: a new tool to examine inflammatory events in cerebral endothelium (June edition, 2005; 85:734-746)', we agree with the fact that the cerebral vasculature shows a high degree of heterogeneity along the vascular tree leading to differential endothelial cell properties, as we discussed in a forthcoming book chapter entitled 'culturing the BBB' in press in 'Blood-Brain Interfaces-From Ontogeny to Artificial Barriers' Dermietzel, Spray, Nedergaard (eds) Wiley-VCH.

Based on many years of experience in Pr Cecchelli's laboratory in the field of the in vitro BBB research, we point out the importance and necessity of using an appropriate isolation procedure that enables us to discard larger vessels, namely arterioles and venules, to obtain a 'capillary-enriched fraction' as we wrote in Coisne et al. ${ }^{1}$ Therefore, we prefer to use the term 'capillary' to distinguish our isolation procedure that enhances a collection of mouse endothelial cells from mainly capillaries, contrary to other procedures that isolate whole brain vessels without using any filtration step to discard macrovessels or that collect mainly microvessels, according to their words. ${ }^{2}$

Concerning the isolation procedure itself, the mechanical homogenization using a Dounce prior to the filtration step remains critical for the success of vessel selection using mesh filtration, as too much or not enough fragmentation of brain vessels with different clearance pestles may modify the homogenate content. Therefore, we take the process of mechanical homogenization into great account in our isolation procedure. Then, contrary to Song and Pachter's protocol that collects whole brain vessels retained on a $75 \mu \mathrm{m}$ mesh surface before a digestion step, we collect the resulting filtrate of the $59 \mu \mathrm{m}$ mesh filtration, which we consider to be more efficient for obtaining mainly capillary fragments (due to their abundance) vs arterioles and venules, and their relative small size.

What is more, the enzymatic digestion of whole brain vessels (retained on a $75 \mu \mathrm{m}$ mesh) prior to their $40 \mu \mathrm{m}$ filtration as described in Song and Pachter's protocol, is debatable. The authors observed that, after vessel digestion, endothelial cells appeared like 'beads on a string'. ${ }^{3}$ This suggests that such a step affects vessel morphology and stability, which may lead to the passage of small vessel fragments or even, endothelial cell clusters, coming from arterioles and/or venules present in these
$75 \mu \mathrm{m}$ mesh surface collected vessels. This is the reason why we prefer to separate larger vessels from capillaries prior to processing for enzymatic digestion, and thus attest that our procedure differs from Song and Pachter's.

Contrary to Dr Pachter's observations, each endothelial cell colony from digested capillaries exhibits the same proliferation rate as the others in our culture conditions. This observation reinforces the fact that our cultures exhibit a homogeneity that could be explained by a common origin in the isolated vessel fragments.

Nevertheless, the most important feature that may attest to the capillary phenotype lays on the BBB characteristics exhibited by endothelial cells in culture, which have been characterized in our in vitro cerebral endothelium. This includes demonstration of tight junction expression and efficiency exhibited by low paracellular permeability, high transendothelial electrical resistance value and $\mathrm{P}$ glycoprotein expression and efficiency, as well as a correlation of the permeability of various compounds to data obtained using a well-characterized and in vivo-correlated bovine in vitro BBB model. ${ }^{4-6}$ In addition to these data, the nonexpression of MECA-32, a mouse antigen known to be downregulated with the establishment of BBB phenotype in capillaries in vivo, reinforces the fact that our cells express a well-differentiated BBB phenotype as observed in vivo and, therefore, provide a suitable support to study the BBB in vitro. Moreover, we were unable to compare our cells to Song and Pachter's as few BBB features have been investigated in their cell culture (Immunostaining and Western blot for Occludin, Claudin-5 and ZO-1) ${ }^{2,3}$

In Coisne et al, ${ }^{1}$ we do not take the expression of von Willebrand factor (VWF) as an evidence of capillary derivation, but as an endothelial marker extensively used to check endothelial cell phenotype in vivo and in vitro without any consideration of the vessel size. We are aware of studies showing a weak or even no expression of VWF in peripheral capillaries in vivo, as mentioned in Dr Pachter's references. However, to our opinion, such concerns cannot provide a strong evidence of the nonbrain capillary origin of our endothelial cell culture as the expression of VWF has already been reported on the BBB in vivo ${ }^{7,8}$ as well as in vitro. ${ }^{3,9}$ Even so, a possible ectopic expression of VWF in vitro could be easily related to culture conditions or the absence of shear stress, as such parameters are well known to influence, to certain extends, endothelial cell phenotype. 
Concerning cell adhesion molecule (CAM) expression on the $\mathrm{BBB}$, numerous authors agree with the fact that CAMs are expressed and modulated by inflammatory stimuli at the BBB. The site of leukocyte extravasation in the brain has not been clearly established. Recent novel investigational technologies as intravital microscopy and microdissection will hopefully contribute to highlight such a process.

To conclude, we are very much concerned by the fact that in vitro BBB models, as all other cell culture models, have as much in the way of inconveniences as advantages. This makes in vitro models interesting only if researchers are aware of model limits. That is why Dr Pachter's comments were welcome and we hope that our answer will serve the in vitro $\mathrm{BBB}$ research community.

Caroline Coisne and Bénédicte Dehouck EA-2465 Pathophysiology of the Blood-Brain Barrier, Université d'Artois-Faculté des Sciences Jean Perrin, Lens Cedex, France

\section{References}

1 Coisne C, Dehouck L, Faveeuw C, et al. Mouse syngenic in vitro blood-brain barrier model: a new tool to examine inflammatory events in cerebral endothelium. Lab Invest 2005;85:734-746.
2 Song L, Pachter JS. Culture of murine brain microvascular endothelial cells that maintain expression and cytoskeletal association of tight junction-associated proteins. In Vitro Cell Dev Biol Anim 2003;39: 313-320.

3 Song L, Pachter JS. Monocyte chemoattractant protein-1 alters expression of tight junction-associated proteins in brain microvascular endothelial cells. Microvasc Res 2004;67:78-89.

4 Cecchelli R, Dehouck B, Descamps L, et al. In vitro model for evaluating drug transport across the bloodbrain barrier. Adv Drug Deliv Rev 1999;36:165-178.

5 Dehouck MP, Meresse S, Delorme P, et al. An easier, reproducible, and mass-production method to study the blood-brain barrier in vitro. J Neurochem 1990; 54:1798-1801.

6 Dehouck MP, Jolliet Riant P, Bree F, et al. Drug transfer across the blood-brain barrier: correlation between in vitro and in vivo models. J Neurochem 1992;58: 1790-1797.

7 Bajaj MS, Kuppuswamy MN, Manepalli AN, et al. Transcriptional expression of tissue factor pathway inhibitor, thrombomodulin and von Willebrand factor in normal human tissues. Thromb Haemost 1999;82: 1047-1052.

8 Gao B, Stieger B, Noe B, et al. Localization of the organic anion transporting polypeptide 2 (Oatp2) in capillary endothelium and choroid plexus epithelium of rat brain. J Histochem Cytochem 1999;47:1255-1264.

9 Unger RE, Oltrogge JB, von Briesen $\mathrm{H}$, et al. Isolation and molecular characterization of brain microvascular endothelial cells from human brain tumors. In Vitro Cell Dev Biol Anim 2002;38:273-281. 